\title{
Using Structure Within Electronic Documents to Make Editors more Accessible
}

\author{
Nick Ayres, Tom Wesley \\ University of Bradford \\ Department of Computing, Bradford, BD7 1DP, United Kingdom \\ Email: t.a.b.wesley@bradford.ac.uk
}

\begin{abstract}
This paper describes on going work within the CAPS ConsortiumCommunication and Access to Information for People with Special Needs, a European Union funded project in the Technology Initiative for Disabled and Elderly People (TIDE) Programme-that is examining ways in which structure within electronic documents may help make those documents more accessible to people with print disabilities. The work concentrates on the production and editing of documents by people with visual impairments and considers how structured editors may be useful to them. A structured editor has been designed to test the ideas, and this is described in the paper.
\end{abstract}

\section{Introduction}

The main work of the CAPS Consortium (Communication and Access to Information for People with Special Needs, a European Union funded project in the Technology Initiative for Disabled and Elderly People (TIDE) Programme) is directed to developing methods to increase the access to information for the print disabled [1]. People with print disabilities include the blind, the deaf-blind, the partially sighted, the dyslexic and those with motor impairments which make it difficult to physically control paper documents.

Accessibility to documents is not just about acquiring information for reading - it also involves the production and editing of documents. Problems are evident here in a number of ways. The first is the creation of a visually interesting document that is often necessary for sighted colleagues. The second problem is created by the limitations imposed upon a visually impaired person by the internal model used by many word processors or editors, and the display of information in a visual or WYSIWYG manner.

Most editors treat the text of a document as a linear character string. By doing so, limitations are placed on the operations that may be performed on documents. For example, documents must be scrolled through in a linear order to reach a particular place. If an electronic document has structure explicitly encoded within it, then additional functionality may be added that is potentially of use to people with print disabilities.

By structure is meant a description of the parts of a document in terms that are independent of the appearance of a document. Thus a document has headings, footnotes and sections, rather than pieces of text with a roman type face indented by a centimetre. When structure is created for electronic documents most existing editors 
specify that individual documents conform to a set of rules. For example, it may be required that a footnote can only appear in a paragraph, and not in a heading. This set of rules is often called a generic document.

A further problem is related to the explosive growth of the graphical user interface (GUI). It is well understood that this poses severe access problems for people with visual impairments. Considerable research and development is underway to provide access to the interface. However, the growth of GUIs is encouraging the growth of visual presentation methods in applications. Structured editors will therefore rely heavily on visual displays of structure. Where this involves actual graphics, this will make it particularly difficult for people with visual impairments to gain the advantages available through the use of structured editors.

The present work has a number of aims. Firstly, to examine what features of structured editors may be of benefit to people with print disabilities. Based on this understanding, it should be possible to produce a set of guidelines to be used in various ways. Potential users may be made more aware of how they can use certain techniques, and hence ask for these in applications, and make choices based on this information. The guidelines should provide information for software producers so that accessibility is built in to their products. Finally the procurers of software within companies will gain assurances that visually impaired people will continue to be able be productive in the commercial office environment, even if this is totally using graphical user interfaces.

\section{Existing Editors and Techniques}

In order to clarify how structure could be useful for people with print disabilities, the literature was examined and a number of structured editbrs were investigated. Full details are presented by Ayres [2]. A number of papers on existing editors and related issues were particularly helpful; these are listed in [3].

Overall, two disadvantages of many of the editors were apparent. Firstly, they were aimed at people who had both a high technical background and also an awareness of the underlying format. Secondly, often few concessions to usability had been made. However, a number of techniques were discussed which could be of use to the print disabled.

\subsection{Selection of Document Units}

Probably the most useful set of techniques are those that allow the selection of a subset of the parts of a document for display and editing. When displayed, these subsets are often called 'views' of a document. Particularly interesting is the document overview, whereby the subset in some way shows the overall organisation of the document. This subset is usually the set of section headings. Conceptually it is possible to use this overview to move the insertion point around the document quicker than having to scroll through the complete text, but this seems to have been rarely implemented.

The overview concept may be extended so that different criteria may be used to select the document units displayed. Examples might be displaying only paragraphs with a particular style or displaying only the lists within a document. This could make it relatively easy to find specific places within a document when such information is 
known. Instead of positive selection criteria, it is possible to have negative selection criteria. Text may be hidden if it meets some criteria, otherwise it is displayed. This mechanism may be used to hide footnotes from the main text of a document.

Having decided which parts of a document to display, editors vary on how the parts are displayed. There are two approaches-display views simultaneously in different windows, or sequentially on request in the same window.

The selection of parts of a document may be used for other purposes than display. Some editors provide a mechanism to select complete units for operations such as cut and paste. This should speed up performance since the units do not bave to be selected by scrolling through the complete text.

\subsection{Creation of Visual Appearance}

Structure is frequently used for the automatic creation of the visual appearance of printed or displayed documents. This presupposes a set of rules that describe transformations from the structure to the visual appearance. In the workplace it is becoming common to have guidelines for the ways in which documents are displayed, that is a corporate style. For example, it may be specified that headings should be in a particular typeface at a particular size. It may possible within structured editors to encode these rules so that a user rarely needs to specify directly the look of a document, but only its structure. If visually impaired writers are able to manipulate the structure, then they may never need to create directly the visual appearance of a document.

\subsection{Automatic Creation of Document Content}

Within many types of documents, there exists content that could be created automatically. These include numbering schemes such as section numbers and footnote numbers. In some cases such as tables of contents and running headers, the text used is often the same as headings. These could be created automatically if an editor was aware of the rules for creating text. This kind of capability is found to a limited extent in one of the subsets of the Open Document Architecture (ODA), FOD36 [4].

Structure may be used to perform automatic numbering of units such as footnotes and sections. While this could be done after editing, numbering can produce useful information to a user about structure within a document. When used as feedback, the interactive numbering system does not have to be the same as that for the final printed document.

\subsection{Feedback Provision}

There are two types of feedback that may be useful-firstly, feedback about the structure created, and secondly, the use of text from structural units to give audio feedback to visually impaired users. Editors use a number of different schemes to provide feedback to the user about the structure that they create. This is often visual in nature, such as different fonts or graphical symbols. Some editors use text labels placed at the start and end of a unit. If the editors were based upon a markup system such as the Standard Generalized Markup Language (SGML), then these labels could 
be the same as that used in the underlying representation, which makes them relatively hard to use.

One of the conclusions of this study is that structure may be used to give meaningful audio feedback to users with visual impairments about operations that they perform. An acknowledged feature of good application design is the use of consistent feedback about operations [5]. There are few things more annoying than the lack of an indication of the result of an operation. For the majority of users, feedback is in visual form and is immediately obvious. Visually impaired users may have to actively check the results of operations to confirm their success. If text associated with specific units, or the name of units operated upon (such as 'footnote'), is given as audio feedback, then visually impaired users will have more immediate feedback about the results of the editing operations.

\subsection{Bookmarks}

A common technique used by structured editors is the bookmark although this is not strictly a feature of structured documents themselves, rather a feature of the editing process. As the name suggests, a bookmark is a mechanism to mark places within documents as being of special interest. At least one system [6] allows a user to give names to bookmarks, which are displayed in a separate window. Users can select the name in order to move to the bookmark or move sequentially through a document to the next or previous bookmark.

\subsection{Creating Structure}

Most structured editors are awkward in the way in which they allow users to indicate the structure in a document. For example, many editors force a user to create a section before being able to create a section heading; an easier technique would have the creation of a section heading signal the creation of a section. This type of awkward interface may be the result of the perceived need to cater for more than one generic document, that is to provide a very general interface. If generic documents are carefully designed, than some of the awkwardness within editors may be removed. As described later, in the current work limitations have been placed on the generic documents handled within the editor in order to remove some of the difficulties for users.

\subsection{The Styles Mechanism}

Early word processors allowed users to specify that particular pieces of text should have properties such as being bold or italic when printed. It was soon realised that when text fulfilled a certain semantic function, for example, a heading, it would often have a set of properties associated with it. Word processors then started to attach a name to this set of properties so that the set could be applied to text in a single operation. This bundling of properties is called the style mechanism [7].

Conceptually styles may be associated with either individual characters, words or paragraphs. Microsoft Word for the Macintosh, for example, has styles that are associated with a complete paragraph, but not with individual characters within a paragraph. 
While examining existing structured editors, it became clear that if constraints are placed upon the rules that a structured document must obey, and styles are associated with structural units, then the styles mechanism may be used to indicate structure within a document. This is being used to a limited extent within the CAPS editor.

\section{The CAPS Editor}

Within the CAPS Project, a prototype structured editor is being produced in order to examine how people with a visual impairment could benefit from some of the ideas discussed in the previous sections.

\subsection{FOD26}

FOD26 [8] is a subset of the Open Document Architecture (ODA) standard [9]. Ayres [10] and Ayres and Wesley [11] have discussed the way ODA may be of value to people with print disabilities. FOD26 provides a relatively simple set of structural types that can be used to test the usefulness for the print disabled of many of the features of structured editors. These include footnotes, sections, automatic numbering of sections and footnotes, styles, alternative textual descriptions of graphics and the automatic generation of a description of how a document will appear visually when printed. This latter capability can be extended with the commercial toolkit produced by the ODA Consortium [12] which also provides a PostScript converter.

As the current investigation is attempting to create general guidelines for the use of structured editors by people with print disabilities, it was decided to use the FOD26 subset of ODA, rather than any proprietary word processor. Quite apart from the difficulty of accessing the internals of proprietary systems, the use of an ISO standard such as ODA allows the investigation of general methods, rather than any particular implementation. The results of the investigation should be valid for structured editors as a whole, and do not depend on the penetration of ODA in the market place.

Recognising that most people use a word processor as an augmented typewriter, it was decided to concentrate on a relatively small number of concepts that are as close to normal usage as possible. It was therefore decided to place limits on the structure within FOD26 that the editor can handle. The style mechanism is being used to input structure. As markers for the start of sections 'inbuilt' heading styles are being used. There are precedents for this in the Word/ODA converter that has been produced by Bull [13].

\subsection{Non-structure Design Decisions}

There were a number of non-structure design issues that had to be made. The ODA Consortium Toolkit can only be used with graphical user interfaces X-Windows on UNIX or MS Windows on PCs. Given the current lack of an effective screen reader for X-Windows, it was decided to base the editor under Windows. However, the state of Windows screen readers leaves much to be desired, so it was also decided to avoid the use of a mouse and that all commands should have hot keys, thus eliminating use of pull down menus. 


\subsection{Document Views}

It was decided to have a number of views of a document. These are the complete document, without footnotes-the normal view; a headings based view-overview and a footnote view that displayed only footnotes. These are displayed as separate windows and switching between views uses a key combination.

The heading overview, besides giving an overall impression of the document organisation, can be used to perform operations on complete sections. By selecting one of the headings, an operation such as cut and paste of complete sections can be performed easily without having to scroll through large amounts of text. The heading is used for audio feedback.

The normal view has a number of interesting features. Structure can be used to ease movement through a document. For example, the insertion point may be moved sequentially from heading to heading. The heading text is also used in this case as feedback. It is possible to move the insertion point to the start or end of paragraphs, in which case the first five or last five words are used as feedback. As a further aid to navigation within a document simple bookmarks are being implemented. While these are not directly visible to a user, it is possible to place and move sequentially between them. This aids quick movement between different places in a document when it is necessary to consult one place while editing text in another.

\subsection{Feedback}

In both the normal view and overview, content is generated to produce feedback about structure. A simple algorithm is used to create section numbers which are displayed within the view to give further feedback about how the sections are organised in the document. This algorithm, used principally to aid the user during document editing, may be different from the one produced when the document is finally formatted and printed.

Another form of feedback is the display of textual labels for units. Examples of this are the use of the name of the style associated with a paragraph, and the placing of the word 'footnote' in the normal view as a place holder for an actual footnote.

\section{Conclusions}

Much effort is placed into making previously created documents accessible for people with print disabilities. However, there are a number of circumstances, such as the work situation, where the accessibility of documents must be considered to include their creation and editing.

The usual model of an electronic document as a character string places limitations upon the editing process; the use of structure adds potential extra functionality that should be able to increase the accessibility of documents for people with visual impairments.

This paper has described some techniques used in existing structured editors that may be adapted for people with a visual impairment. A structured editor that is specifically aimed at testing the use of these techniques has been designed and is being implemented, in order to produce a set of guidelines that may be used within commercial editors. 


\section{Acknowledgement}

This work has been partly funded by DGXIII of the Commission of the European Union, under its Technology Initiative for Disabled and Elderly People (TIDE) Programme. The other CAPS partners are the Royal National Institute for the Blind (UK), Sensotec BV (BE), Infovox (SE) and the Handicap Institute (SE).

\section{References}

1. Full details of the CAPS Consortium can be obtained from the Coordinator, Professor Jan Engelen at the Katholieke Universiteit, Leuven, Belgium. The Consortium maintains an ftp site, gate.esat.kuleuven.ac.be in the directory /pub/CAPS and its sub directories, which provides access to its latest public documents.

2. N. Ayres: Structured Editors-Existing Systems and Techniques Report. CAPS Internal Report, August 1993. Obtainable from the Coordinator [1]

3. D.D. Chamberlin, H.F. Hasselmeier, A.W. Luniewski, D.P. Paris, B.W. Wade, M.L. Zolliker: Quill: An Extensible System for Editing Documents of Mixed Type. In: 21st International Conference on System Science. Hawaii: 1988, pp. $317-326$

Complexity in Structured Documents: User Interface Issues. In: R. Furuta (ed.): International Conference on Electronic Publishing, Document Manipulation and Typography EP90. Gaithersburg: CUP 1990, pp. 79-91

ISO/IEC TR 10037 (E) 1991 Information technology-SGML and Text-entry Systems-Guidelines for SGML Syntax-Directed Editing Systems. International Organisation for Standardisation

M.D.P. Leland, R.S. Fish, R.E. Kraut: Collaborative Document Production using Quilt. In: Conference on Computer Supported Cooperative Work. Portland Oregon: 1988

V. Quint, I. Vatton: Grif: An Interactive System for Structured Document Manipulation. In: J.C. van Vliet (ed.): Text Processing and Document Manipulation. Nottingham: CUP 1986

V. Quint: Systems for the Manipulation of Structured Documents. In: J. Andre et al. (ed.): Structured Documents. CUP 1989, pp. 39-74

V. Quint, I. Vatton: Combining Hypertext and Structured Documents in Grif. In: D. Lucarella et al. (ed.): Proceedings of the ACM Conference on Hypertext. Milano: ACM Press 1992, pp. 23-32

A. Vercoustre: Structured Editing-Hypertext Approach: Cooperation and Complementarity. In: R. Furuta (ed.): International Conference on Electronic Publishing, Document Manipulation and Typography EP90. Gaithersburg: CUP 1990, pp. 65-78

J.H. Walker, R.L. Bryan: An Editor for Structured Technical Documents. In: J.H. Miller (ed.): Protext IV. Boston, USA: Boole 1987, pp. 145-150 J.H. Walker: Supporting Document Development with Concordia. Computer 23, 48-55 1988

4. ISO/IEC DISP 111821992 Information Technology-International Standardized Profile FOD36 - Office Document Format - Extended document structure Character, Raster Graphics and Geometric Graphics content architectures - Part 1: Document Application Profile 
5. A. Dix, J. Finlay, G. Abowd, R. Beale: Human Computer Interaction. Prentice Hall 1993, pp. 131-145

6. J.H. Walker: The Role of Modularity in Document Authoring Systems. In: ACM Conference on Document Processing Systems. Santa Fe, New Mexico: 1988, pp. $117-124$

7. J. Johnson, R.J. Beach: Styles in Document Editing Systems. IEEE Computer 23, 32-43 1988

8. ISO/IEC DISP 11181-1 1991 Information Technology-International Standardized Profile FOD26 - Office Document Format - Enhanced document structure - Character, raster graphics and geometric graphics content architectures - Part 1: Document Application Profile. International Organisation for Standardisation

9. ISO 86131988 Information processing - Text and Office systems-Office Document Architecture (ODA) and Interchange Formats. International Organisation for Standardisation

H. Brown: Introduction to the Office Document Architecture. In: J. Rosenberg (ed.): Multi-media Document Translation ODA and the EXPRES Project. Springer Verlag 1991

10. N. Ayres: ODA-State of the Art Report. CAPS internal report, February 1993. Obtainable from the Coordinator [1]

11. N. Ayres, T. Wesley: Open Document Architecture-An Opportunity for Information Access for the Print Disabled. In: The 3rd International Conference on Computers for Handicapped Persons. Vienna 1992, pp. 556-561 N. Ayres, T. Wesley: Automated Braille Production from ODA Documents. In: European Conference on the Advancement of Rehabilitation Technology, ECART 2. Stockholm: 1993

12. ODA Consortium Toolkit, ODA Consortium, Avenue Marcel Thiry 204, B-1200 Brussels

13. Bull ODA Product Set Users Guide, Bull SA, March 1992 